\title{
ESTUDIO EXPERIMENTAL DEL SECADO A TEMPERATURAS CONVENCIONALES DE ACACIAS
}

Experimental study of wood drying at conventional-temperature of Acacias

\section{Rubén A. ANANIAS ${ }^{1}$, Linette SALVO ${ }^{1}$, Ricardo ESTRADA ${ }^{1}$, Rodrigo BRIONES ${ }^{2}$}

${ }^{1}$ Depto. Ingeniería en Maderas. Fac. Ingeniería. Universidad del Bío-Bío. Concepción. Chile.

2División Industrias. Instituto Forestal.rbriones@infor.cl

Autor para correspondencia: ananias@ubiobio.cl

Recibido: 07.01.2008. Aceptado: 12.07. 2008.

\section{RESUMEN}

Entre las especies del género Acacias, la Acacia melanoxylon o aromo australiano y la Acacia dealbata o aromo chileno, son dos especies aclimatadas en Chile con un interesante potencial industrial. En este trabajo, se estudian programas de secado a temperaturas convencionales y la calidad de la madera seca de A. melanoxylon y A. dealbata.

Experimentalmente son realizados 8 ensayos de secado a temperaturas convencionales, usando un horno de secado convectivo de $0.3 \mathrm{~m}^{3}$ de capacidad. El diseño experimental incluye como factores variables la especie (A. melanoxylon y A. dealbata), el espesor de la madera $(25$ y $50 \mathrm{~mm})$ y el tipo de corte (tangencial y radial).

Los resultados indican que para 25 o $50 \mathrm{~mm}$ de espesor, la $A$. melanoxylon y la $A$. dealbata, en corte radial y tangencial se dejan secar satisfactoriamente bajo un mismo programa de temperaturas convencionales. La calidad de la madera seca se ve favorecida por la carencia de colapso y la baja anisotropía de la contracción transversal.

Palabras clave: calidad de secado, contracción, Acacia melanoxylon, Acacia dealbata.

\begin{abstract}
Acacia melanoxylon and Acacia dealbata are two Australian species well acclimated in Chile, both wood species with an interesting industrial potential. In this work, has been studied kiln drying runs and the wood drying quality of the sawn wood of Acacia melanoxylon and Acacia dealbata during kiln drying at conventional temperatures.

Eight tests of kiln drying to conventional temperatures are made, using a convective drying of $0,3 \mathrm{~m}^{3}$ of capacity. The experimental design includes as variable the species (A. melanoxylon an A. dealbata), the wood thickness $(25$ and $50 \mathrm{~mm}$ ) and the cut-type (flatsawn-wood and quartersawn-wood). The wood drying quality on the basis of European and Chilean standards is evaluated.
\end{abstract}

The results show that for a same thickness, both species A. melanoxylon and A. dealbata, in flatsawn 
and quartersawn-wood could be satisfactorily kiln dryied under a similar program of conventional temperatures. The wood drying quality is performed due to both species are free of collapse and they are an low transversal shrinkage.

Keywords: drying quality, shrinkage, Acacia melanoxylon, Acacia dealbata

\section{INTRODUCCIÓN}

El aromo australiano (Acacia melanoxylon) y aromo chileno (Acacia dealbata) son dos especies del género Acacias que se han adaptado rápidamente en el territorio chileno. Estas especies son similares en cuanto a sus propiedades anatómicas y físicas. Respecto de su estructura anatómica presentan grandes vasos generalmente solitarios o en bandas radiales de 2 a 3 vasos, parénquima longitudinal paratraqueal vasicéntrico y radios leñosos multiseriados heterogéneos (Figura 1).

En relación a sus propiedades físicas muestran pequeñas diferencias en la magnitud de la densidad básica, la contracción transversal y la anisotropía de la contracción transversal, siendo la $A$. dealbata de mayor densidad básica, contracción y anisotropía transversal, que la $A$. melanoxylon (Tabla 1). Estos valores son comparables a los reportados en la literatura (INFOR-CONAF 1998, Ananías 1989).

Tabla 1: Propiedades físicas de las Acacias (Fuente: Estrada et al. 2004)

\begin{tabular}{|l|c|c|c|c|}
\hline \multirow{2}{*}{ Especie } & \multirow{2}{*}{ CHmax } & \multirow{2}{*}{$\begin{array}{c}\text { Sog } \\
(\%)\end{array}$} & Ctg (\%) & Crd (\%) \\
\cline { 4 - 5 } & $\left(\mathrm{kg} / \mathrm{m}^{3}\right)$ & \multicolumn{2}{|c|}{$\mathrm{CH}=12 \%$} \\
\hline Acacia melanoxylon & 177 & 430 & 4.2 & 3.6 \\
\hline Acacia dealbata & 161 & 470 & 5.0 & 4.4 \\
\hline
\end{tabular}




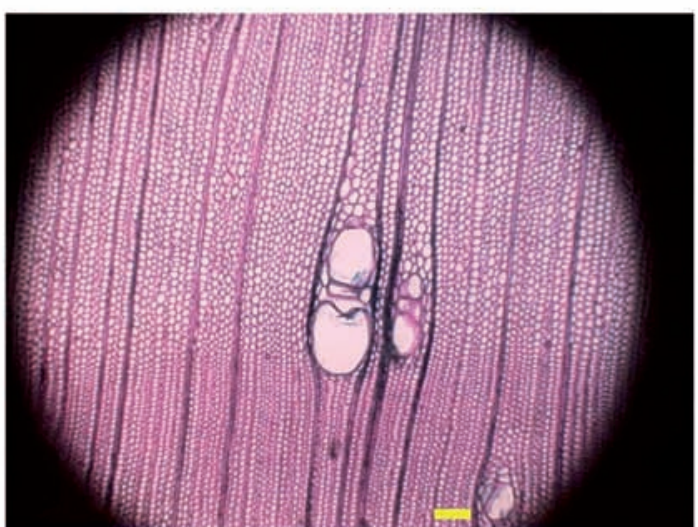

a) Acacia dealbata (transversal 100x-50 um)

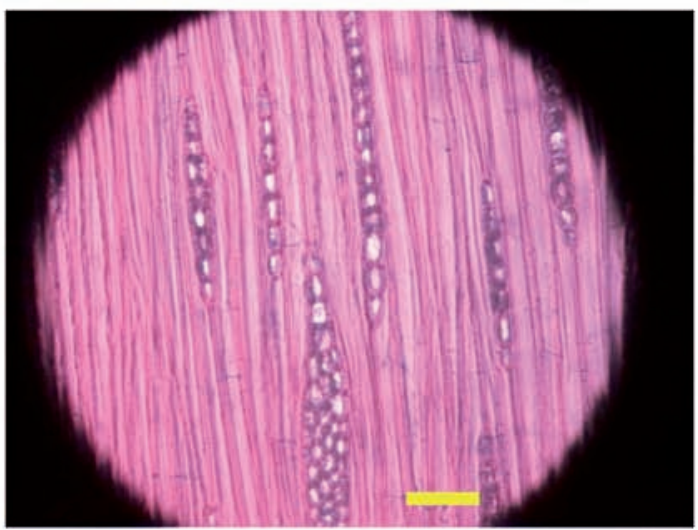

c) Acacia dealbata (tangencial $400 \mathrm{x}-50 \mathrm{um}$ )

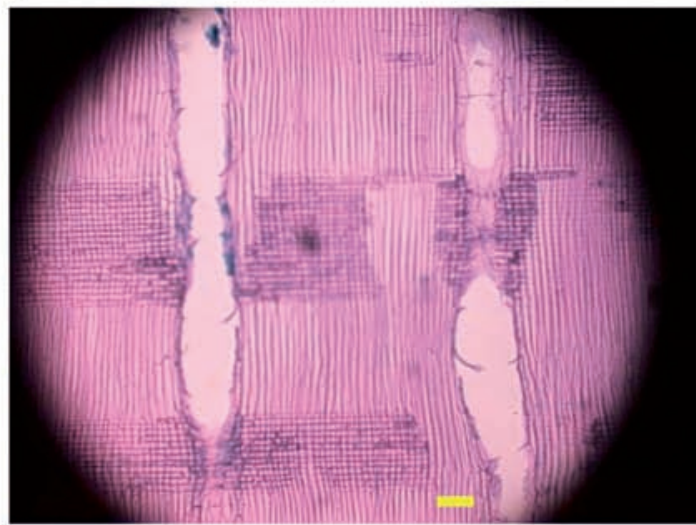

e) Acacia dealbata (radial 100x-50 um)

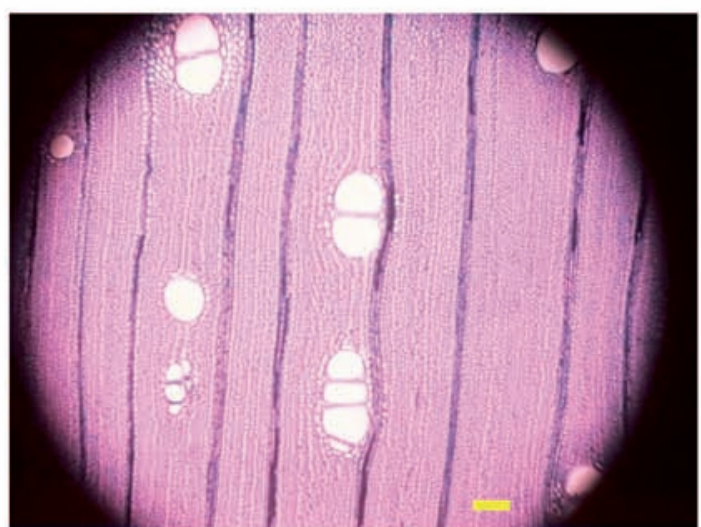

b) Acacia melanoxylon (transversal 100x-50 um)

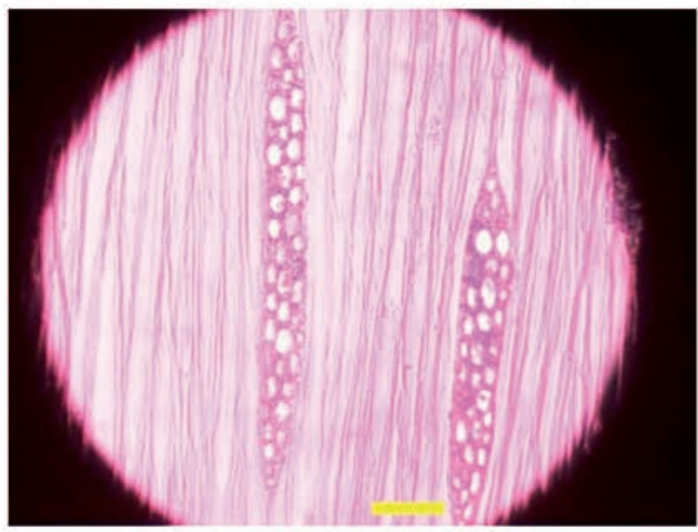

d) Acacia melanoxylon (tangencial 400x-50 um)

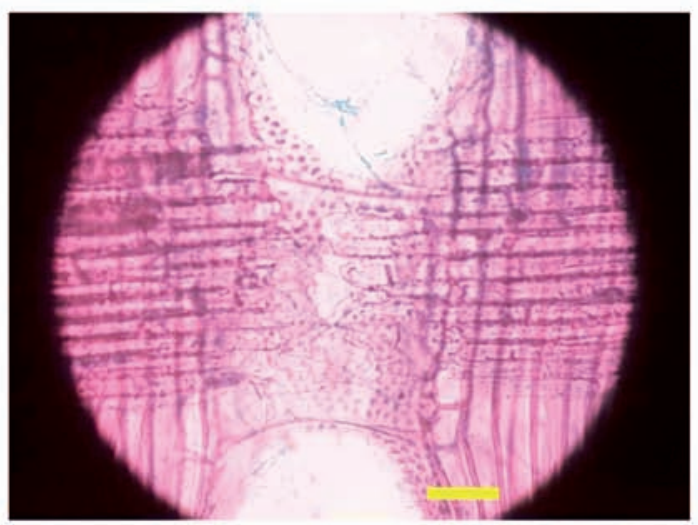

f) Acacia melanoxylon (radial 400x-50 um)

Figura 1. Muestras microtomadas de las Acacias (Fuente: Burgos y Requena, 2005; Palma y Montero 2005) 
Respecto del secado de Acacias, en Chile, se ha reportado el secado por alta temperatura de Acacia melanoxylon con buenos resultados, particularmente para la madera presecada al aire (Ananías et al. 1996; Fuentes, 1993). Igualmente en Nueva Zelanda los mejores resultados para el secado de Acacia melanoxylon, se han encontrado con una combinación de presecado hasta $30 \%$ de contenido de humedad y un secado final al horno a temperatura convencionales (Haslett 1986, 1983; Nicholas y Brown 2002). La Acacia dealbata ha sido reportada como de más fácil secado en comparación a la Acacia melanoxylon (Chudnoff, 1984).

En este trabajo se estudian programas de secado convencional y la calidad de la madera seca para Acacia melanoxylon y Acacia dealbata.

\section{METODOLOGIA}

La madera utilizada para estos ensayos es obtenida de bosques artificiales creciendo en la Octava Región de Chile. Las trozas fueron convertidas en madera aserrada en 25 o $50 \mathrm{~mm}$ de espesor en época de verano y almacenada bajo techo en un galpón, desde donde fue preparada a 1 metro de largo antes de los ensayos de secado. Los ensayos de secado fueron realizados en un horno de secado piloto de $0.3 \mathrm{~m}^{3}$ de capacidad. La carga fue armada con 96 piezas de $25 \mathrm{~mm}$ de espesor, esto es, 7 piezas en el ancho y 8 corridas de altura, para $50 \mathrm{~mm}$ la carga se armó con 60 piezas, o sea, 6 piezas en el ancho y 10 camadas en altura. En todos los ensayos se utilizó una velocidad del aire de $3 \mathrm{~m} / \mathrm{s}$, con cambio de giro cada 6 horas; separadores secos, cepillados y libres de defectos de $20 \mathrm{~mm}$ de espesor (Figura 2).
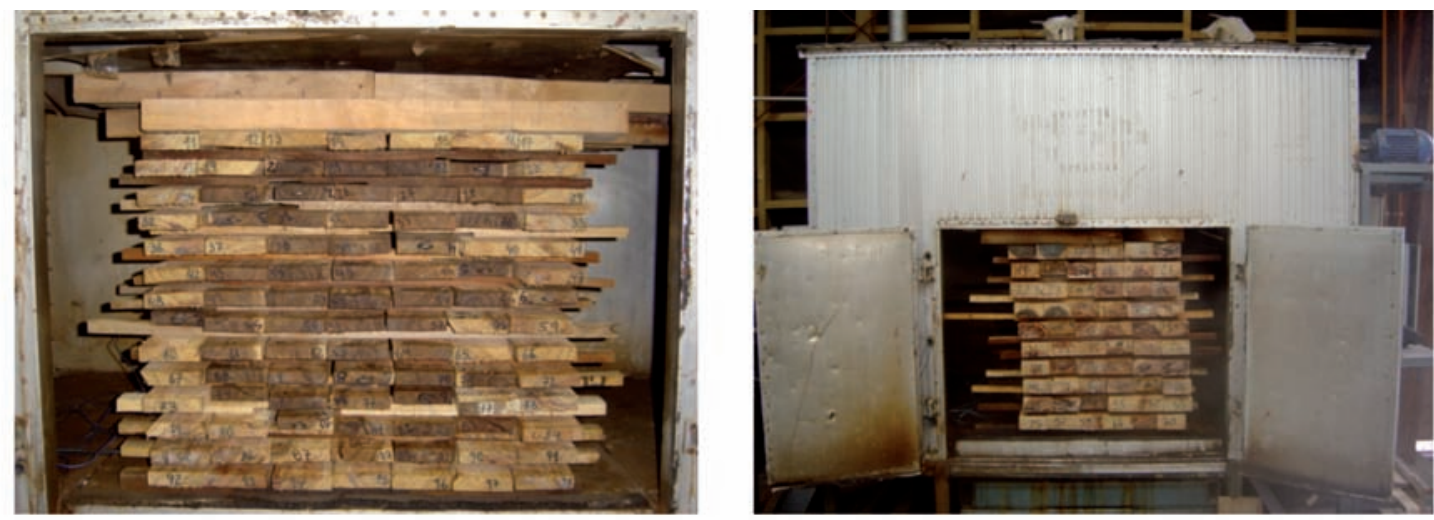

Figura 2: Organización de la madera de 25 y $50 \mathrm{~mm}$ en el horno experimental

Fueron realizados 8 ensayos de secado, correspondientes a la combinación: especie (A. dealbata y $A$. melanoxylon), espesor (25 y $50 \mathrm{~mm}$ ) y tipo de corte (tangencial y radial).

La calidad del secado fue evaluada a través de: heterogeneidad de la humedad final, gradiente de humedad, tensiones de secado y colapso, usando las estandarizaciones europeas según tablas 2 y 3 (Welling 1994; Pérez et al. 2007).

Tabla 2: Especificaciones para evaluar la calidad del secado

\begin{tabular}{|c|c|c|c|}
\hline Estándar & CHF (\%) & GH (\%) & TFS (mm) \\
\hline 1 & $7-13$ & 4 & 3 \\
\hline 2 & $8-12$ & 3 & 2 \\
\hline 3 & $9-11$ & 2 & 1 \\
\hline
\end{tabular}

1:calidad estándar. 2: calidad secado. 3: calidad exclusiva CHF: contenido humedad final. GH: gradiente humedad. TFS: tensiones finales de secado 
Tabla 3: Intensidad de las grietas y colapso

\begin{tabular}{|c|c|c|c|c|}
\hline Intensidad & GI (\%) & GS (mm) & GE (cm) & CO (mm) \\
\hline Severa & $>10$ & $>5$ & $>20$ & $>6$ \\
\hline Moderada & $2-10$ & $2-5$ & $5-20$ & $3-6$ \\
\hline Leve & $<2$ & $<2$ & $<5$ & $<3$ \\
\hline
\end{tabular}

GI:grietas internas. GS: grietas superficiales. GE: grietas extremos. CO: colapso

Además se evaluaron los alabeos de acuerdo a las recomendaciones de Kauman y Mittak 1966, Pérez et al. 2007 y la NCh 993 (tablas 4 y 5).

Tabla 4: Niveles de alabeos admisibles en mm adaptados para madera de

$1 \mathrm{~m}$ de largo de la norma NCH 993.

\begin{tabular}{|c|c|c|c|c|c|}
\hline Alabeo & Espesor & S/alabeo & Leve & Moderado & Intenso \\
\hline Acanaladura & \multirow{4}{*}{25} & 0 & $1-3$ & $3-5$ & $>5$ \\
\hline Arqueadura & & 0 & $1-3$ & $3-6$ & $>6$ \\
\hline Encorvadura & & 0 & $1-2$ & $2-3$ & $>3$ \\
\hline Torcedura & & 0 & $1-2$ & $2-7$ & $>7$ \\
\hline Acanaladura & \multirow{4}{*}{50} & 0 & $1-3$ & $3-5$ & $>5$ \\
\hline Arqueadura & & 0 & $0-1$ & $1-3$ & $>3$ \\
\hline Encorvadura & & 0 & $1-4$ & $4-7$ & $>7$ \\
\hline Torcedura & & 0 & $1-2$ & $2-7$ & $>7$ \\
\hline
\end{tabular}

Tabla 5: Niveles de alabeos admisibles en mm adaptados para madera de $2.4 \mathrm{~m}$ de largo de la norma NCH 993.

\begin{tabular}{|c|c|c|c|c|c|}
\hline Alabeo & Espesor & S/alabeo & Leve & Moderado & Intenso \\
\hline Acanaladura & \multirow{4}{*}{25} & 0 & $1-3$ & $3-5$ & $>5$ \\
\hline Arqueadura & & 0 & $1-17$ & $17-33$ & $>33$ \\
\hline Encorvadura & & 0 & $1-3$ & $3-6$ & $>6$ \\
\hline Torcedura & & 0 & $1-10$ & $10-18$ & $>18$ \\
\hline Acanaladura & \multirow{4}{*}{50} & 0 & $1-3$ & $3-5$ & $>5$ \\
\hline Arqueadura & & 0 & $1-9$ & $9-17$ & $>17$ \\
\hline Encorvadura & & 0 & $1-3$ & $3-6$ & $>6$ \\
\hline Torcedura & & 0 & $1-4$ & $4-8$ & $>8$ \\
\hline
\end{tabular}

La humedad de la madera durante el secado fue determinada mediante pesadas de muestras testigos. Inicialmente se determinó la humedad de la madera a través de probetas que fueron secadas en estufa por 24 horas a $103 \pm 2{ }^{\circ} \mathrm{C}$ hasta peso constante, luego se calculó la masa anhidra de 10 muestras testigo con el fin de obtener la humedad por pesadas de tales muestras en el transcurso del secado. La evolución temporal de las temperaturas de bulbo seco y bulbo húmedo en el interior del horno así como la temperatura en el centro de la madera, fueron monitoreadas usando termocuplas tipo K, los datos fueron almacenados en un sistema de adquisición de datos.

\section{RESULTADOS}

Evolución del contenido de humedad y la temperatura de la madera y del ambiente

La evolución de las condiciones de humedad y temperaturas de la madera y el ambiente obtenidas en los 8 ensayos de secado son mostradas en las Figuras 3 y 4 . 


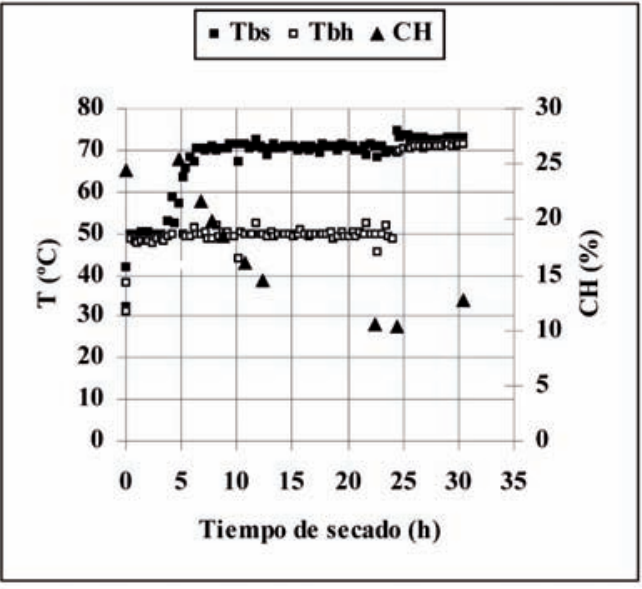

A. melanoxylon, tangencial

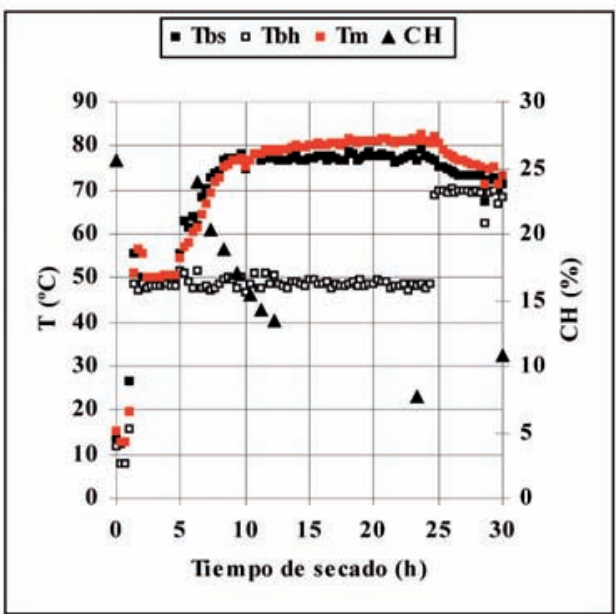

A. dealbata, tangencial

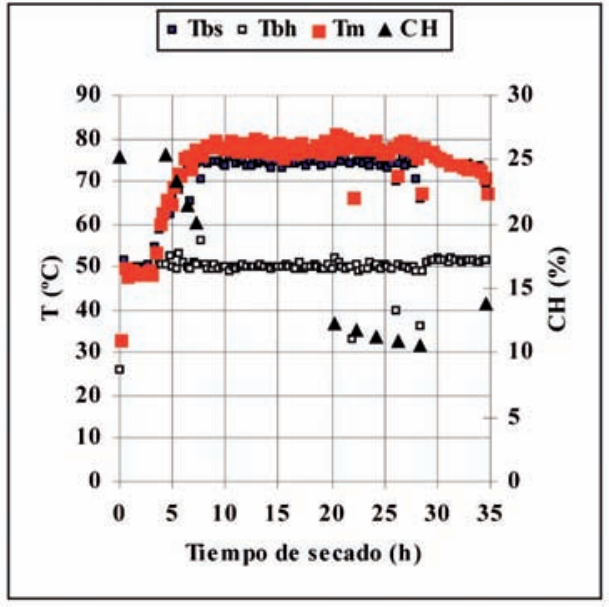

A. melanoxylon, radial

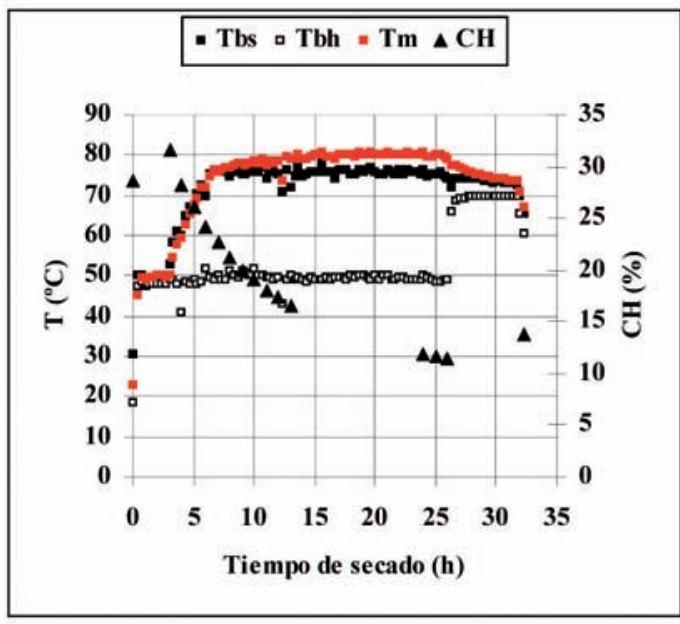

A. dealbata, radial

Figura 3. Curvas de secado Acacias de $25 \mathrm{~mm}$ 


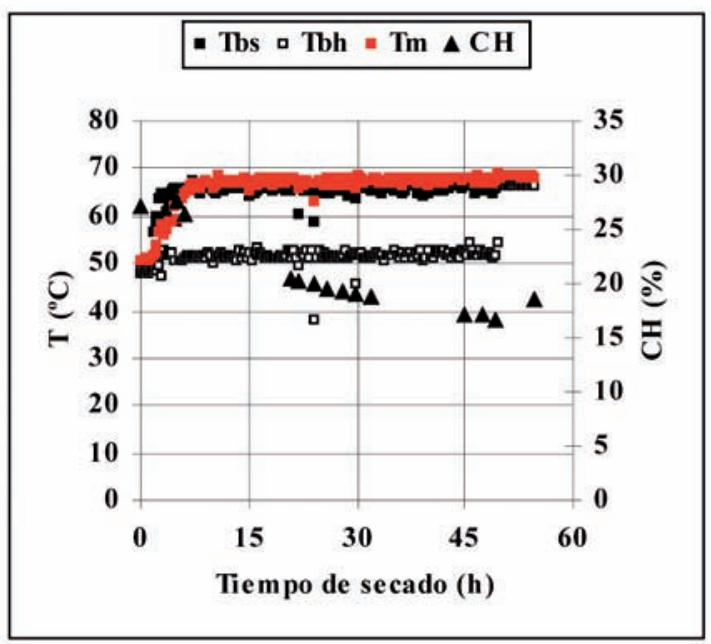

A. melanoxylon, tangencial

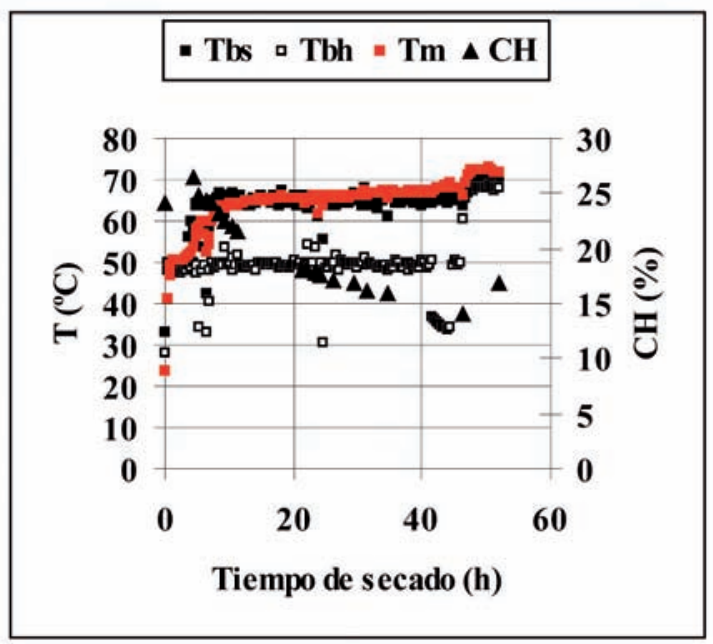

A. dealbata, tangencial

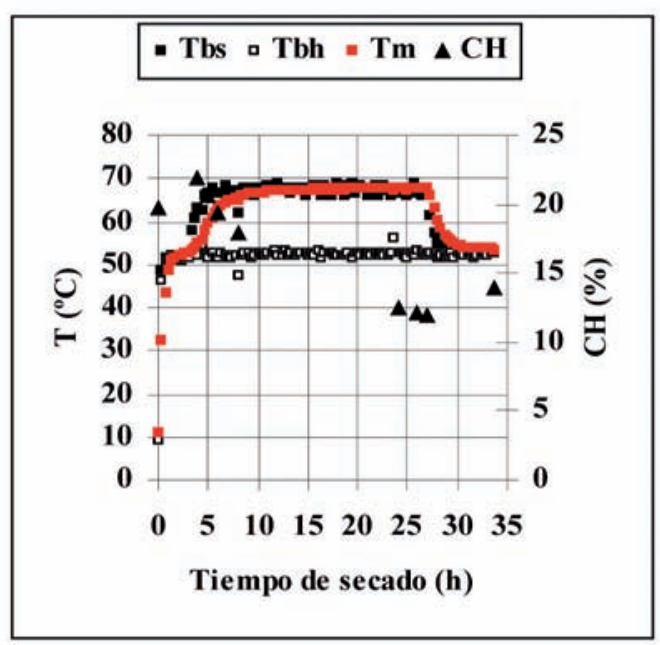

A. melanoxylon, radial

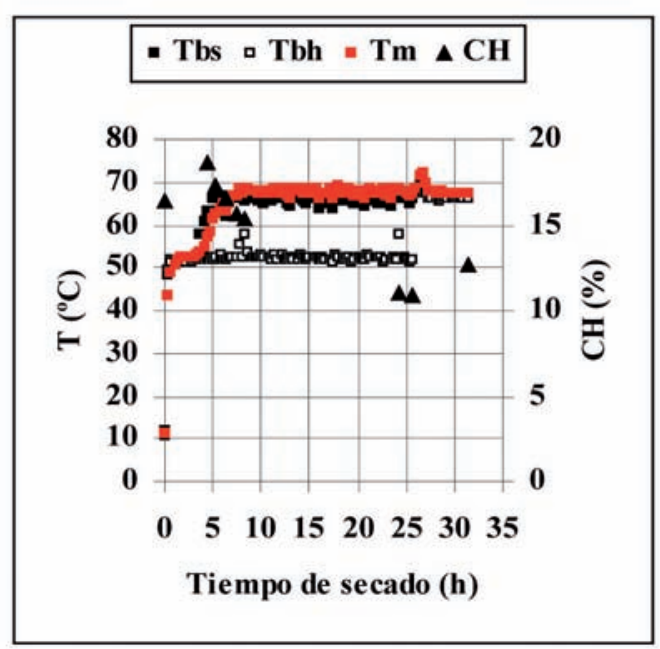

A. dealbata, radial

Figura 4. Curvas de secado Acacias de $50 \mathrm{~mm}$

Como la madera fue almacenada bajo cobertizo en época estival antes del secado final, la humedad inicial en los ensayos de secado al horno varía entre 25 y 30 \%. Este almacenamiento actuó como un presecado. Por lo mismo el tiempo de secado es reducido a entre 30 y 50 horas para la madera de 25 y $50 \mathrm{~mm}$ de espesor respectivamente.

A pesar del reducido tiempo de secado, la madera seca no presenta grietas internas ni colapso y la contracción volumétrica transversal es debajo de 5\% (Figura 5). La magnitud de la contracción volumétrica transversal durante estos ensayos de secado a temperaturas convencionales, como era de esperar, es menor que el 7\% de contracción transversal volumétrica obtenida durante el secado por alta temperatura $\left(110 / 70{ }^{\circ} \mathrm{C} /{ }^{\circ} \mathrm{C}\right)$ de madera oreada de Acacia melanoxylon de $25 \mathrm{~mm}$ de espesor reportado por Ananías et al. 1996. 


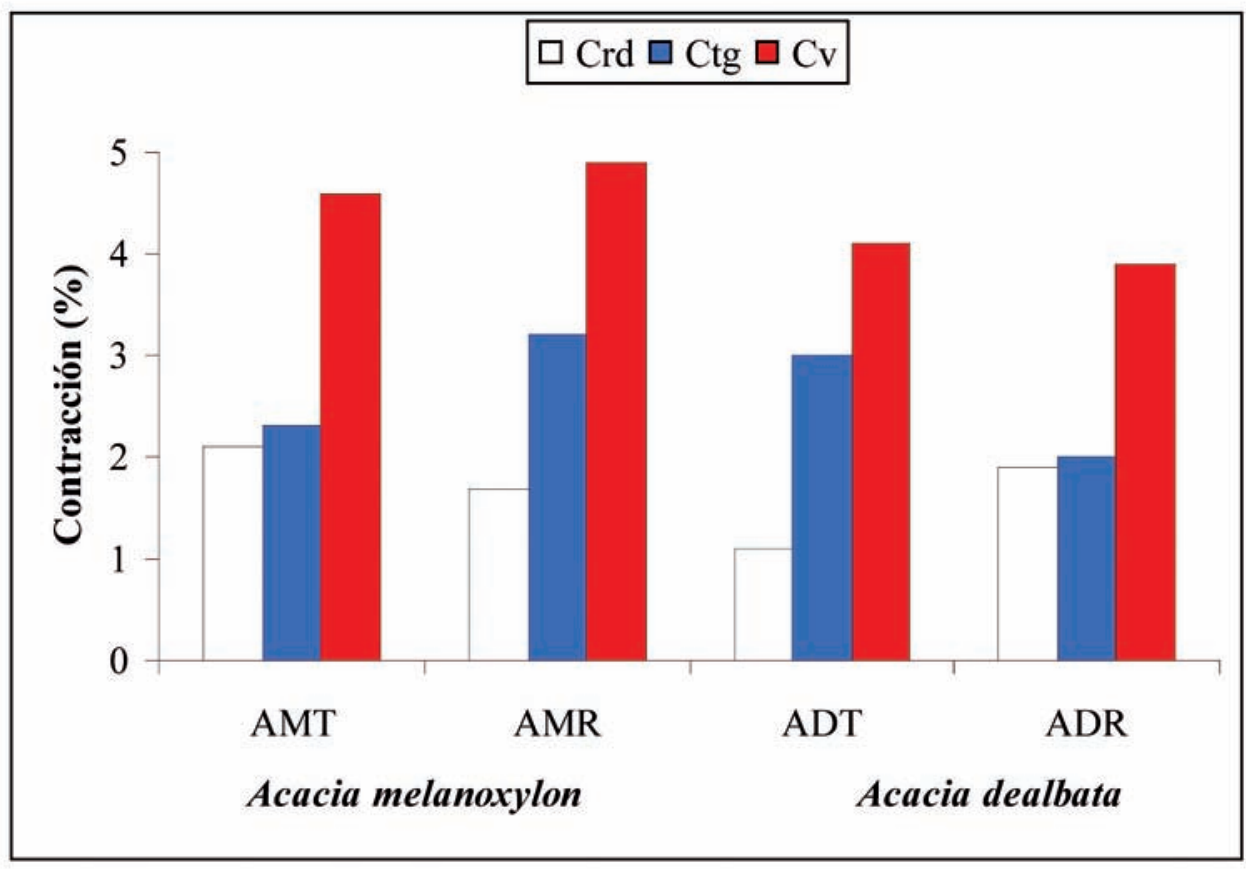

a) $25 \mathrm{~mm}$

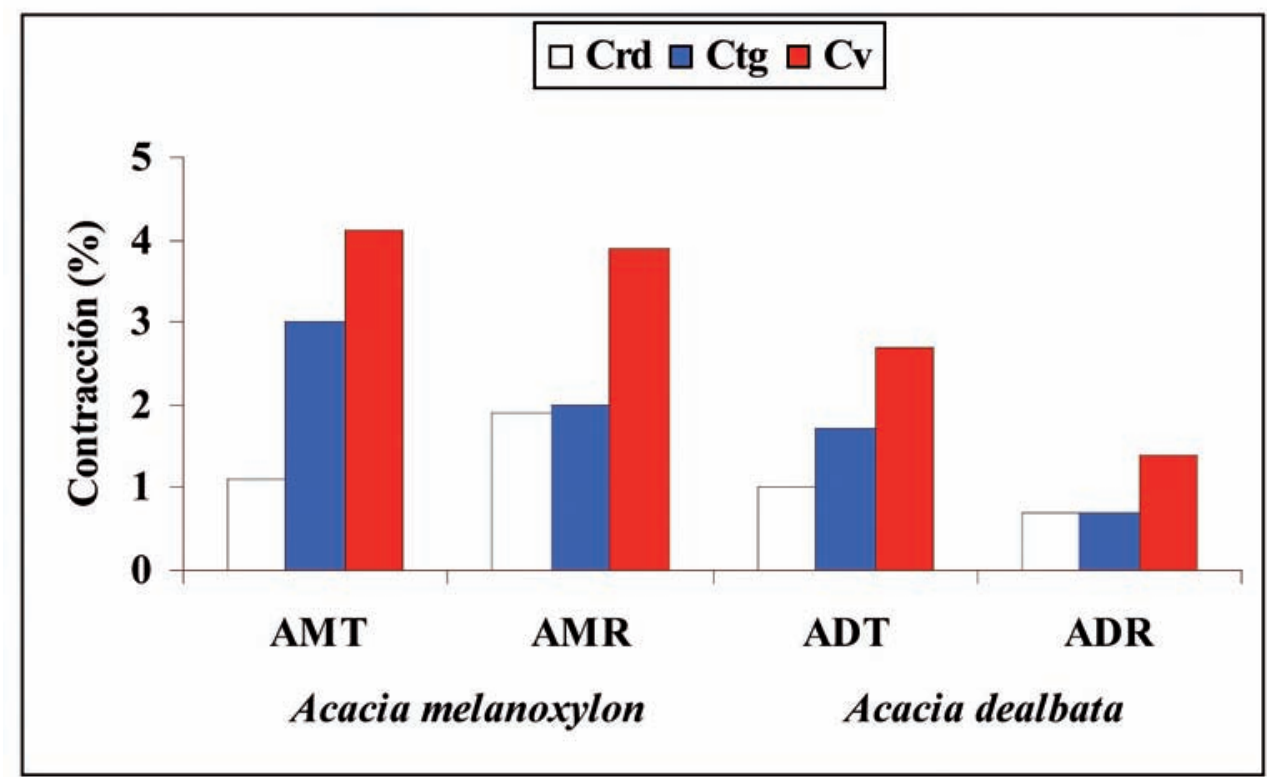

b) $50 \mathrm{~mm}$

Figura 5: Contracción al final del secado de Acacias

$\mathrm{Crd}=$ contracción radial $\mathrm{Ctg}=$ contracción tangencial. $\mathrm{Cv}=$ contracción volumétrica $\mathrm{AMT}=$ Acacia melanoxylon en corte tangencial. $\mathrm{AMR}=$ Acacia melanoxylon en corte radial $\mathrm{ADT}=$ Acacia dealbata en corte tangencial. $\mathrm{ADR}=$ Acacia dealbata en corte radial 
La carencia de grietas y colapso, en la madera presecada de A. dealbata y A. melanoxylon, esta probablemente relacionada con la presencia de grandes vasos, carentes de tilosis, que favorecen el movimiento del agua durante el secado, así como con la baja contracción y anisotropía de la contracción transversal, observados en la Figura 5. La carencia de grietas internas y colapso en estos ensayos de secado a temperatura convencional también había sido observado durante ensayos de secado por alta temperatura de Acacia melanoxylon (Ananías et al. 1996)

Por otra parte, la calidad del secado piloto en base a las condiciones de humedad y tensiones al final del secado se muestran en la Figura 6. El contenido de humedad final y el gradiente de humedad final presentan mayor heterogeneidad y la mayor parte de madera es clasificada en calidad estándar. Respecto de las tensiones, en todos los ensayos en corte tangencial se observa un 100\% de la madera en el rango de calidad exclusiva

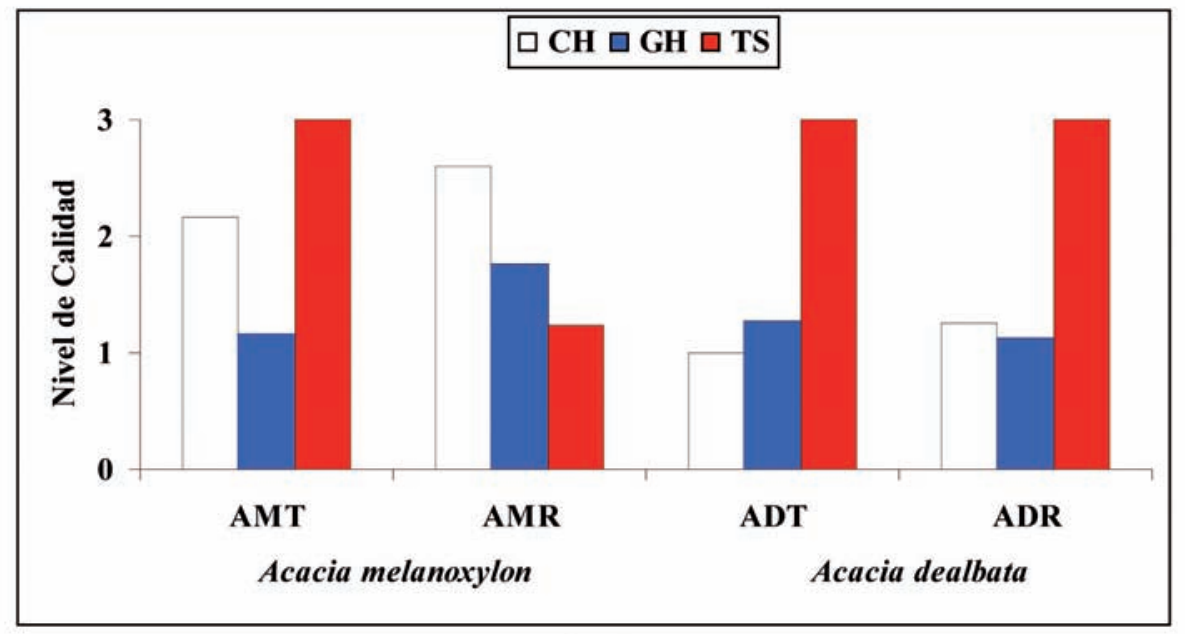

a) $25 \mathrm{~mm}$

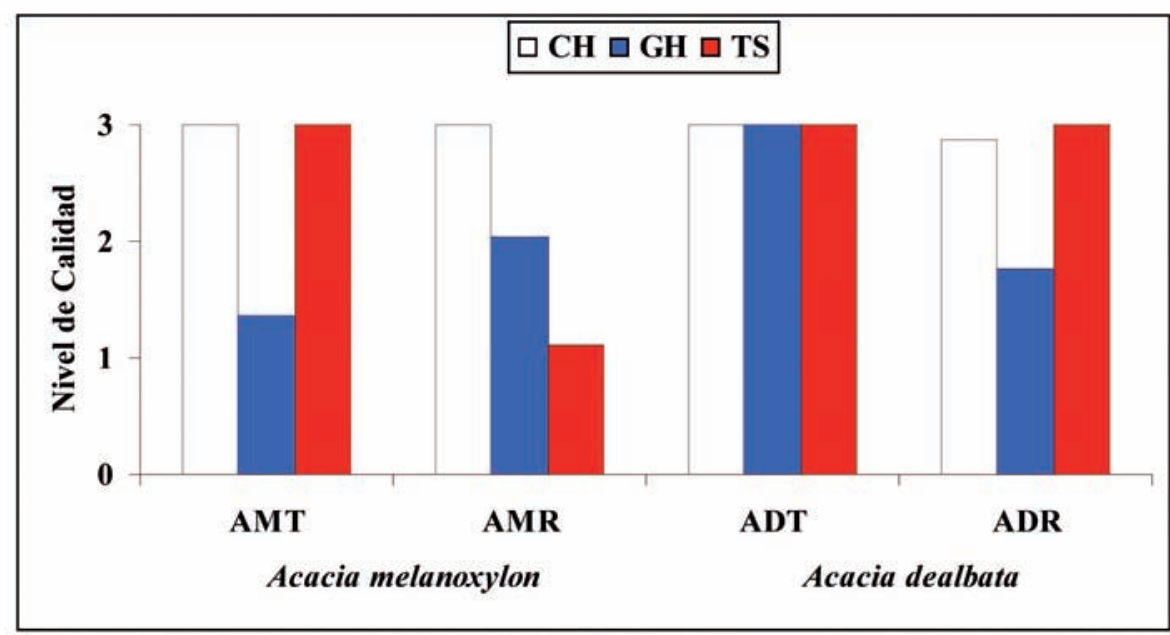

b) $50 \mathrm{~mm}$

Figura 6: Nivel de calidad según rango estandarizado para humedad final $(\mathrm{CH})$, gradiente humedad (GH) y tensiones (TS) al final del de secado.

$\mathrm{AMT}=$ Acacia melanoxylon en corte tangencial. $\mathrm{AMR}=$ Acacia melanoxylon en corte radial $\mathrm{ADT}=$ Acacia dealbata en corte tangencial. $\mathrm{ADR}=$ Acacia dealbata en corte radial 
Los alabeos recogidos durante los ensayos de secado se presentan en la Figura 7

Figura 7: Intensidad de los alabeos

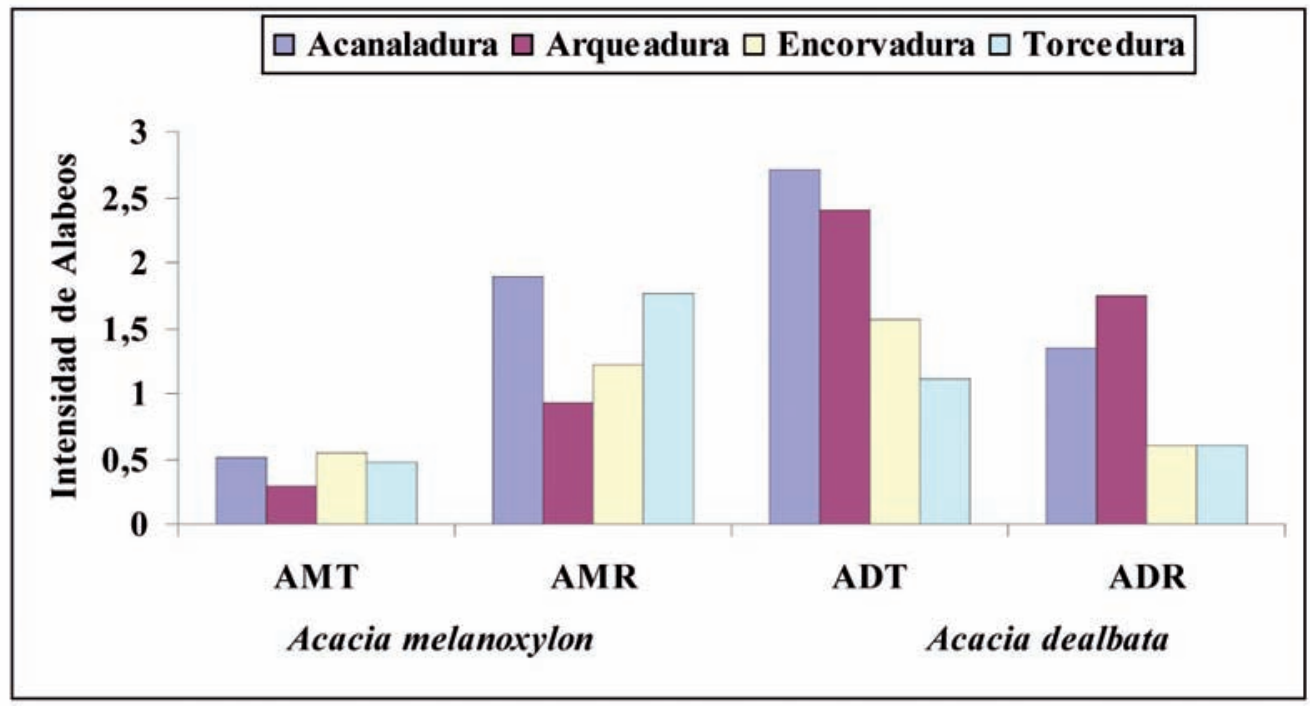

a) $25 \mathrm{~mm}$

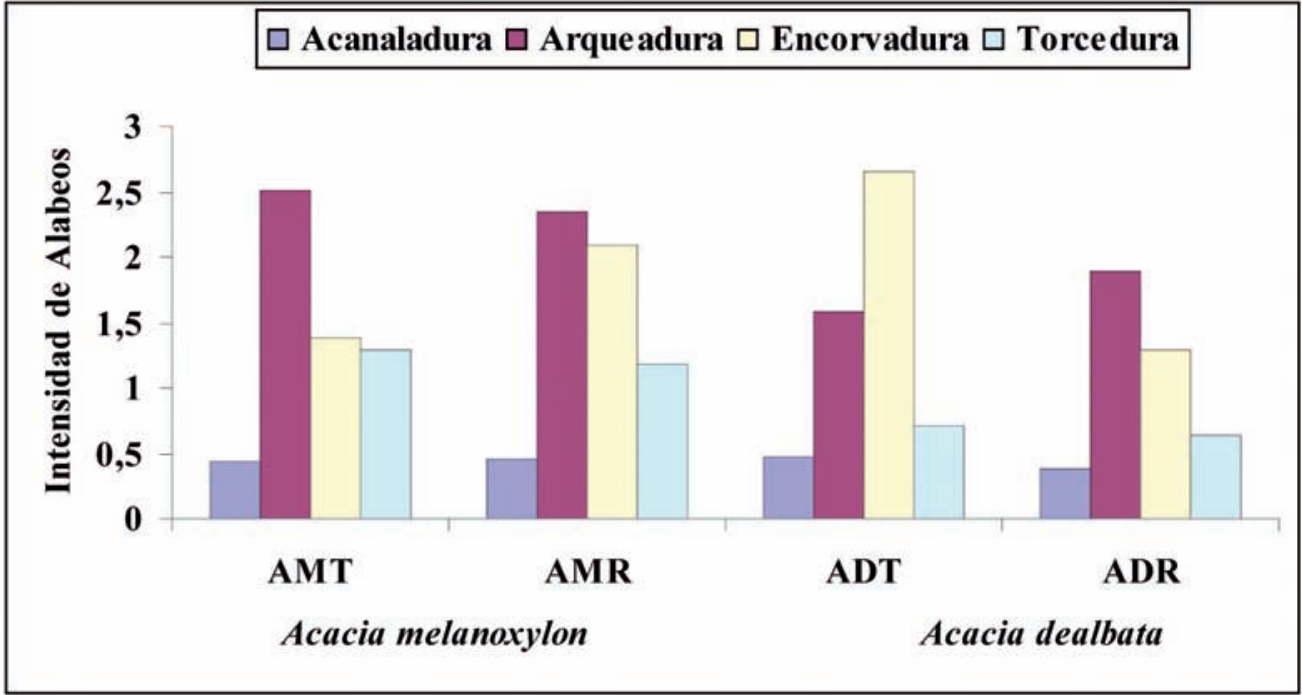

b) $50 \mathrm{~mm}$

$\mathrm{AMT}=$ Acacia melanoxylon en corte tangencial. $\mathrm{AMR}=$ Acacia melanoxylon en corte radial $\mathrm{ADT}=$ Acacia dealbata en corte tangencial. $\mathrm{ADR}=$ Acacia dealbata en corte radial

Los alabeos más intensos en ambas especies son acanaladura, encorvadura y arqueadura. En general las Acacias de $50 \mathrm{~mm}$ de espesor, presentan una mayor intensidad de alabeos en comparación a la madera de $25 \mathrm{~mm}$ de espesor. La Acacia melanoxylon en corte tangencial y la Acacia dealbata en corte radial son las que presentan menores niveles de alabeos en 25 y $50 \mathrm{~mm}$ de espesor. La arqueadura y encorvadura son los alabeos más intensos en ambas Acacias de $50 \mathrm{~mm}$ de espesor. Coincidentemente, Ananías et al. 1996, reporta que la arqueadura y la encorvadura fueron los alabeos más intensos durante el secado por alta temperatura de Acacia melanoxylon. 


\section{Programas de secado}

En el cuadro 6 se proponen los programas de secado para Acacia melanoxylon y Acacia dealbata en 25 y $50 \mathrm{~mm}$ de espesor. La propuesta esta basada en los resultados experimentales observados inicialmente y en base de la literatura (Ananías et al. 1996, Simpson, 1996, Fuentes, 1993; Rozsa, 1993; Boone et al. 1988; Haslett, 1986 ).

Cuadro 6: Programas de secado para Acacia melanoxylon y Acacia dealbata.

\begin{tabular}{|c|c|c|c|c|}
\hline Espesor (mm) & $\mathbf{C H ~ ( \% )}$ & $\mathbf{T}\left({ }^{\mathbf{0}} \mathbf{C}\right)$ & $\mathbf{T}_{\mathbf{H}}\left({ }^{\mathbf{0}} \mathbf{C}\right)$ & $\mathbf{C H E}(\mathbf{\%})$ \\
\hline \multirow{3}{*}{25} & $30-25$ & 50 & 50 & - \\
\cline { 2 - 5 } & $25-10$ & 75 & 50 & 4 \\
\cline { 2 - 5 } & 10 & 75 & 75 & - \\
\hline \multirow{3}{*}{50} & $30-25$ & 50 & 50 & - \\
\cline { 2 - 5 } & $25-10$ & 65 & 50 & 6 \\
\cline { 2 - 5 } & 10 & 65 & 65 & - \\
\hline
\end{tabular}

$\mathrm{CH}$ : contenido de humedad. T: temperatura del bulbo seco. $\mathrm{T}_{\mathrm{H}}$ : temperatura bulbo húmedo. CHE: contenido de humedad de equilibrio

Se propone un único programa de temperaturas de secado, comenzando a un contenido de humedad debajo del $30 \%$, dependiente del espesor e independiente del tipo de corte y especie. Ambos programas incluyen un calentamiento inicial a $50{ }^{\circ} \mathrm{C}$, a continuación una fase de secado constante $75 / 50\left({ }^{\circ} \mathrm{C} /{ }^{\circ} \mathrm{C}\right) \mathrm{o}$ $65 / 50\left({ }^{\circ} \mathrm{C} /{ }^{\circ} \mathrm{C}\right)$ para 25 y $50 \mathrm{~mm}$ de espesor respectivamente, para terminar con 6 horas de acondicionado final en ambiente saturado a $75^{\circ} \mathrm{C}$ o $65^{\circ} \mathrm{C}$ según el espesor de la madera.

\section{CONCLUSIONES}

Los resultados indican que para 25 o $50 \mathrm{~mm}$ de espesor, la $A$. melanoxylon y la $A$. dealbata, en corte radial y tangencial se dejan secar satisfactoriamente bajo un mismo programa de temperaturas convencionales. La calidad de la madera seca se ve favorecida por la carencia de colapso, una baja anisotropía de la contracción transversal y una reducida intensidad de tensiones de secado y alabeos.

\section{AGRADECIMIENTOS}

Al Instituto Forestal de Chile (INFOR) en particular al proyecto FDI/CORFO "Masificación y desarrollo de opciones productivas en base a especies de Acacia probadas en Chile", por permitir la divulgación de estos resultados.

\section{BIBLIOGRAFIA}

Ananías, R.A.; Flores, P.; Fuentes, O.; Peña, G. 1996. Secado por alta temperatura de latifoliadas. Actas VIII Reunión de investigación y desarrollo en productos forestales 143-154. Universidad de Chile, Santiago, Chile.

Ananías, R.A. 1989. Variabilidad de la densidad básica y la contracción en madera de aromo australiano. Ciencia e Investigación Forestal 6(6):119-130. 
Boone, R.S.; Kozlik, C.J.; Bois, P.J.; Wengert, E.M. 1988. Dry kiln schedules for commercial woods. Temperate and tropical. USDA, FPL-GTR-57.

Burgos, R.; Requena, J. 2005. Microscopía de aromo chileno. Informe Laboratorio de Anatomía de la Madera. Ingeniería Civil en Industrias Forestales, Departamento de Ingeniería en Maderas, Facultad de Ingeniería, Universidad del Bío-Bío. 16 pp.

Chudnoff, M. 1984. Tropical timbers of the world. USDA Forest Service. Agriculture Handbook $\mathrm{N}^{\circ}$ 607.

Estrada, B.; Fernández, J.; Prádenas, C. 2004. Propiedades físicas del aromo australiano y aromo chileno. Informe Laboratorio de Propiedades Físicas de la Madera. Ingeniería Ejecución en Maderas, Departamento de Ingeniería en Maderas, Facultad de Ingeniería, Universidad del Bío-Bío. 13 pp.

Fuentes, O. 1993. Secado por alta temperatura: Ensayos sobre madera oreada de aromo australiano de 30 mm de espesor. Seminario Titulación, Departamento de Ingeniería en Maderas, Facultad de Ingeniería, Universidad del Bío-Bío, Concepción, Chile.

Haslett, A.N. 1986. Properties and utilisation of exotic specialty timbers grown in New Zealand. Part II: Australian Blackwood Acacia melanoxylon R.Br. New Zealand Forest Service, FRI, Bulletin 119. 12p.

Haslett, A.N. 1983. Drying properties of New Zealand-grown Acacia melanoxylon. New Zealand Journal of Forestry Science 13(2):130-138.

INFOR-CONAF. 1998. Monografía de aromo australiano. Instituto Forestal, santiago, Chile. 81 pp

INN. 1993. Norma chilena NCH 993 EOf 72. Madera. Procedimientos y criterios de evaluación para clasificación. Instituto nacional de normalización. Santiago, Chile.

Kauman, W.; Mittak, G. 1966. Ensayos de secado de coigüe. Informe Técnico Nº 25, INFOR.

Nicholas, I.; Brown, I. 2002. Blackwood: A handbook for growers and end users. Forest Research Bulletin $\mathrm{N}^{\circ}$ 225, FRI, Rotorua, New Zealand.

Palma, C.; Montero, C. 2005. Microscopía de aromo australiano. Informe Laboratorio de Anatomía de la Madera. Ingeniería Civil en Industrias Forestales, Departamento de Ingeniería en Maderas, Facultad de Ingeniería, Universidad del Bío-Bío. 14 pp

Pérez, P.; Ananías, R.A.; Hernández, G. 2007. Estudio experimental del secado de renovales de canelo Drimys winteri. Maderas. Ciencia y tecnología 9(1):59-70.

Rozsa, A. 1993. Drying native hardwoods for high quality end use. Report FP312, CSIRO, Melbourne. Australia.

Simpson, W. 1996. Method to estimate dry-kiln schedules and species grouping. Tropical and temperate hardwoods. USDA, FPL- RP N 548.

Welling, J. 1994. Drying quality assessment and specification. A challenge for the future. $4^{\text {th }}$ IWDC: 297-304. 